\title{
A SIMPLE QUANTUM EQUATION FOR DECOHERENCE through interaction with the environment
}

\author{
Erasmo Recami \\ Facoltà di Ingegneria, Università statale di Bergamo, Dalmine, Italy; \\ and INFN - Sezione di Milano, Milan, Italy. \\ recami@mi.infn.it \\ Ruy H. A. Farias \\ LNLS - Synchrotron Light National Laboratory; Campinas, S.P.; Brazil. \\ ruy@lnls.br
}

\begin{abstract}
Within the density matrix formalism, it is shown that a simple way to get decoherence is through the introduction of a "quantum" of time (chronon): thus replacing the differential Liouville-von Neumann equation with a finite-difference version of it. In this way, one is given the possibility of using a rather simple quantum equation to describe the decoherence effects due to interaction with the environment.

Namely, the mere introduction (not of a "time-lattice", but simply) of a "chronon" allows us to go on from differential to finite-difference equations; and in particular to write down the Scroedinger equation (as well as the Liouvillevon Neumann equation) in three different ways: "retarded", "symmetrical", and "advanced". One of such three formulations - the retarded one- describes in an elementary way a system which is exchanging (and losing) energy with the environment. In its density-matrix version, indeed, it can be easily shown that all non diagonal terms go to zero very rapidly. [A much larger presentation of this work appeared c/o the Lanl Archives as the e-print quant-ph/9706059, and as the preprint IC/98/74, ICTP; Trieste, 1998].
\end{abstract}

keywords: quantum decoherence, interaction with the environment, quantum measurement theory, finite-difference equations, chronon, Caldirola, density-matrix formalism, Liouville-von Neumann equation, proceedings 


\section{Introduction}

In this note, we briefly mention the consequences of the introduction of a quantum of time $\tau_{0}$ in the formalism of non-relativistic quantum mechanics, by referring ourselves, in particular, to the theory of the chronon as proposed by P.Caldirola. Let us recall that such an interesting "finite difference" theory, forwards — at the classical level-a solution for the motion of a particle endowed with a non-negligible charge in an external electromagnetic field, overcoming all the known difficulties met by Abraham-Lorentz's and Dirac's approaches (and even allowing a clear answer to the question whether a free falling charged particle does or does not emit radiation), and - at the quantum levelyields a remarkable mass spectrum for leptons.

In unpublished work [cf. the e-print quant-ph/9706059, and the subsequent Report IC/98/74 (I.C.T.P.; Trieste, 1998), where also extensive references can be found], after having reviewed Caldirola's approach, we [1] worked out, discussed, and compared to one another the new representations of Quantum Mechanics (QM) resulting from it, in the Schrödinger, Heisenberg and density-operator (Liouville-von Neumann) pictures, respectively.

Let us stress that, for each representation, three (retarded, symmetric and advanced) formulations are possible, which refer either to times $t$ and $t-\tau_{0}$, or to times $t-\tau_{0} / 2$ and $t+\tau_{0} / 2$, or to times $t$ and $t+\tau_{0}$, respectively. It is interesting to notice that, when the chronon tends to zero, the ordinary QM is obtained as the limiting case of the "symmetric" formulation only; while the "retarded" one does naturally appear to describe QM with friction, i.e., to describe dissipative quantum systems (like a particle moving in an absorbing medium). In this sense, discretized QM is much richer than the ordinary one.

In the mentioned unpublished work [1], we have also obtained the (retarded) finitedifference Schrödinger equation within the Feynman path integral approach, and studied some of its relevant solutions. We have then derived the time-evolution operators of this discrete theory, and used them to get the finite-difference Heisenberg equations. 7 Afterwards, we have studied some typical applications and examples: as the free particle, the harmonic oscillator and the hydrogen atom; and various cases have been pointed out, for which the predictions of discrete QM differ from those expected from "continuous" QM. We want here to give our attention to the fact that, when applying the density matrix formalism to the solution of the measurement problem in QM, very interesting results are met, as, for instance, a natural explication of "decoherence" [2]: which seem to reveal the power of dicretized (in particular, retarded) QM.

${ }^{*}$ When discussing therein the mutual compatibility of the various pictures listed above, we found that they can actually be written in a form such that they result to be equivalent (as it happens in the "continuous" case of ordinary QM), even if the Heisenberg picture cannot be derived by "discretizing" directly the ordinary Heisenberg representation. 


\section{Outline of the classical approach}

If $\rho$ is the charge density of a particle on which an external electromahnertic field acts, the famous Lorentz's force law

$$
\mathbf{f}=\rho\left(\mathbf{E}+\frac{\mathbf{1}}{\mathbf{c}} \mathbf{v} \wedge \mathbf{B}\right)
$$

is valid only when the particle charge $q$ is negligible with respect to the external field sources. Otherwise, the classical problem of the motion of a (non-negligible) charge in an electromegnetic field is still an open question. For instance, after the known attempts by Abraham and Lorentz, in 1938 Dirac[3] obtained and proposed his famous classical equation

$$
m \frac{\mathrm{d} u_{\mu}}{\mathrm{d} s}=F_{\mu}+\Gamma_{\mu}
$$

where $\Gamma_{\mu}$ is the Abraham 4-vector

$$
\Gamma_{\mu}=\frac{2}{3} \frac{e^{2}}{c}\left(\frac{\mathrm{d}^{2} u_{\mu}}{\mathrm{d} s^{2}}+\frac{u_{\mu} u^{\nu}}{c^{2}} \frac{\mathrm{d}^{2} u_{\nu}}{\mathrm{d} s^{2}}\right)
$$

that is, the (Abraham) reaction force acting on the electron itself; and $F_{\mu}$ is the 4 -vector that represents the external field acting on the particle

$$
F_{\mu}=\frac{e}{c} F_{\mu \nu} u^{\nu}
$$

At the non-relativistic limit, Dirac's equation goes formally into the one previously obtained by Abraham-Lorentz:

$$
m_{0} \frac{\mathrm{d} \mathbf{v}}{\mathrm{d} t}-\frac{2}{3} \frac{e^{2}}{c^{3}} \frac{\mathrm{d}^{2} \mathbf{v}}{\mathrm{d} t^{2}}=e\left(\mathbf{E}+\frac{1}{c} \mathbf{v} \wedge \mathbf{B}\right) .
$$

The last equation shows that the reaction force equals $\frac{2}{3} \frac{e^{2}}{c^{3}} \frac{\mathrm{d}^{2} \mathbf{v}}{\mathrm{d} t^{2}}$.

Dirac's dynamical equation (2) presents, however, many troubles, related to the infinite many non-physical solutions that it possesses. Actually, it is a third-order differential equation, requiring three initial conditions for singling out one of its solutions. In the description of a free electron, e.g., it yields "self-accelerating" solutions (runaway solutions), for which velocity and acceleration increase spontaneously and indefinitely. Moreover, for an electron submitted to an electromagnetic pulse, further non-physical solutions appear, related this time to pre-accelerations: If the electron comes from infinity with a uniform velocity $v_{0}$ and, at a certain instant of time $t_{0}$, is submitted to an electromagnetic pulse, then it starts accelerating before $t_{0}$. Drawbacks like these motivated further attempts to find out a coherent (not pointlike) model for the classical electron.

Considering elementary particles as points is probably the sin plaguing modern physics (a plague that, unsolved in classical physics, was transferred to quantum physics). One of 
the simplest way for associating a discreteness with elementary particles (let us consider, e.g., the electron) is just via the introduction (not of a "time-lattice", but merely) of a "quantum" of time, the chronon, following Caldirola. [4] Like Dirac's, Caldirola's theory is also Lorentz invariant (continuity, in fact, is not an assumption required by Lorentz invariance). This theory postulates the existence of a universal interval $\tau_{0}$ of proper time, even if time flows continuously as in the ordinary theory. When an external force acts on the electron, however, the reaction of the particle to the applied force is not continuous: The value of the electron velocity $u_{\mu}$ is supposed to jump from $u_{\mu}\left(\tau-\tau_{0}\right)$ to $u_{\mu}(\tau)$ only at certain positions $s_{n}$ along its world line; these "discrete positions" being such that the electron takes a time $\tau_{0}$ for travelling from one position $s_{\mathrm{n}-1}$ to the next one $s_{n}$. The electron, in principle, is still considered as pointlike, but the Dirac relativistic equations for the classical radiating electron are replaced: (i) by a corresponding finite-difference (retarded) equation in the velocity $u^{\mu}(\tau)$

$$
\begin{array}{r}
\frac{m_{0}}{\tau_{0}}\left\{u_{\mu}(\tau)-u_{\mu}\left(\tau-\tau_{0}\right)+\frac{u_{\mu}(\tau) u_{\nu}(\tau)}{c^{2}}\left[u_{\nu}(\tau)-u_{\nu}\left(\tau-\tau_{0}\right)\right]\right\}= \\
=\frac{e}{c} F_{\mu \nu}(\tau) u_{\nu}(\tau),
\end{array}
$$

which reduces to the Dirac equation (2) when $\tau_{0} \rightarrow 0$; and (ii) by a second equation [the transmission law] connecting this time the discrete positions $x^{\mu}(\tau)$ along the world line of the particle:

$$
x_{\mu}\left(n \tau_{0}\right)-x_{\mu}\left[(n-1) \tau_{0}\right]=\frac{\tau_{0}}{2}\left\{u_{\mu}\left(n \tau_{0}\right)-u_{\mu}\left[(n-1) \tau_{0}\right]\right\},
$$

which is valid inside each discrete interval $\tau_{0}$, and describes the internal motion of the electron. In these equations, $u^{\mu}(\tau)$ is the ordinary four-vector velocity, satisfying the condition $u_{\mu}(\tau) u^{\mu}(\tau)=-c^{2}$ for $\tau=n \tau_{0}$, where $n=0,1,2, \ldots$ and $\mu, \nu=0,1,2,3$; while $F^{\mu \nu}$ is the external (retarded) electromagnetic field tensor, and the chronon associated with the electron (by comparison with Dirac's equation) resulted to be

$$
\frac{\tau_{0}}{2} \equiv \theta_{0}=\frac{2}{3} \frac{k e^{2}}{m_{0} c^{3}} \simeq 6.266 \times 10^{-24} \mathrm{~s},
$$

depending, therefore, on the particle (internal) properties [namely, on its charge $e$ and rest mass $\left.m_{0}\right]$.

As a result, the electron happens to appear eventually as an extended-like[5] particle, with internal structure, rather than as a pointlike object. For instance, one may imagine that the particle does not react instantaneously to the action of an external force because of its finite extension (the numerical value of the chronon is of the same order as the time spent by light to travel along an electron classical diameter). As already said, eq.(6) describes the motion of an object that happens to be pointlike only at discrete positions $s_{n}$ along 
its trajectory; even if both position and velocity are still continuous and well-behaved functions of the parameter $\tau$, since they are differentiable functions of $\tau$. It is essential to notice that a discreteness character is given in this way to the electron without any need of a "model" for the electron. Actually it is well-known that many difficulties are met not only by the strictly pointlike models, but also by the extended-type particle models ("spheres", "tops", "gyroscopes", etc.). We deem the answer stays with a third type of models, the "extended-like" ones, as the present approach; or as the (related) theories [5] in which the center of the pointlike charge is spatially distinct from the particle center-ofmass. Let us repeat, anyway, that also the worst troubles met in quantum field theory, like the presence of divergencies, are due to the pointlike character still attributed to (spinning) particles; since - as we already remarked - the problem of a suitable model for elementary particles was transported, unsolved, from classical to quantum physics. One might say that problem to be the most important even in modern particle physics. Equations (6) and the following one, together, provide a full description of the motion of the electron; but they are free from pre-accelerations, self-accelerating solutions, and problems with the hyperbolic motion.

In the non-relativistic limit the previous (retarded) equations get simplified, into the form

$$
\begin{aligned}
& \frac{m_{0}}{\tau_{0}}\left[\mathbf{v}(t)-\mathbf{v}\left(t-\tau_{0}\right)\right]=e\left[\mathbf{E}(t)+\frac{1}{c} \mathbf{v}(t) \wedge \mathbf{B}(t)\right], \\
& \mathbf{r}(\mathbf{t})-\mathbf{r}\left(\mathbf{t}-\tau_{\mathbf{0}}\right)=\frac{\tau_{0}}{2}\left[\mathbf{v}(\mathbf{t})-\mathbf{v}\left(\mathbf{t}-\tau_{\mathbf{0}}\right)\right],
\end{aligned}
$$

The important point is that eqs.(6), or eqs.(7), allow to overcome the difficulties met with the Dirac classical equation. In fact, the electron macroscopic motion is completely determined once velocity and initial position are given. The explicit solutions of the above relativistic-equations for the radiating electron - or of the corresponding non-relativistic equations - verify that the following questions cab be regarded as having been solved within Caldirola's theory: A) exact relativistic solutions: 1) free electron motion; 2) electron under the action of an electromagnetic pulse; 3) hyperbolic motion; B) nonrelativistic approximate solutions: 1) electron under the action of time-dependent forces;

2) electron in a constant, uniform magnetic field; 3) electron moving along a straight line under the action of an elastic restoring force.

In refs. [1] we studied the electron radiation properties as deduced from the finite-difference relativistic equations (6), and their series expansions, with the aim of showing the advantages of the present formalism w.r.t. the Abraham-Lorentz-Dirac one.

\subsection{The three alternative formulations}

Two more (alternative) formulations are possible of Caldirola's equations, based on different discretization procedures. In fact, equations (6) and (7) describe an intrinsically 
radiating particle. And, by expanding equation (6)) in terms of $\tau_{0}$, a radiation reaction term appears. Caldirola called those equations the retarded form of the electron equations of motion.

On the contrary, by rewriting the finite-difference equations in the form:

$$
\begin{gathered}
\frac{m_{0}}{\tau_{0}}\left\{u_{\mu}\left(\tau+\tau_{0}\right)-u_{\mu}(\tau)+\frac{u_{\mu}(\tau) u_{\nu}(\tau)}{c^{2}}\left[u_{\nu}\left(\tau+\tau_{0}\right)-u_{\nu}(\tau)\right]\right\}= \\
=\frac{e}{c} F_{\mu \nu}(\tau) u_{\nu}(\tau) \\
x_{\mu}\left[(n+1) \tau_{0}\right]-x_{\mu}\left(n \tau_{0}\right)=\tau_{0} u_{\mu}\left(n \tau_{0}\right)
\end{gathered}
$$

one gets the advanced formulation of the electron theory, since the motion is now determined by advanced actions. At variance with the retarded formulation, the advanced one describes an electron which absorbs energy from the external world.

Finally, by adding together retarded and advanced actions, Caldirola wrote down the symmetric formulation of the electron theory:

$$
\begin{gathered}
\frac{m_{0}}{2 \tau_{0}}\left\{u_{\mu}\left(\tau+\tau_{0}\right)-u_{\mu}\left(\tau-\tau_{0}\right)+\frac{u_{\mu}(\tau) u_{\nu}(\tau)}{c^{2}}\left[u_{\nu}\left(\tau+\tau_{0}\right)-u_{\nu}\left(\tau-\tau_{0}\right)\right]\right\}= \\
=\frac{e}{c} F_{\mu \nu}(\tau) u_{\nu}(\tau) \\
x_{\mu}\left[(n+1) \tau_{0}\right]-x_{\mu}\left((n-1) \tau_{0}\right)=2 \tau_{0} u_{\mu}\left(n \tau_{0}\right)
\end{gathered}
$$

which does not include any radiation reactions, and describes a non radiating electron.

Before closing this brief introduction to the classical "chronon theory", let us recall at least one more result derivable from it. If we consider a free particle and look for the "internal solutions" of the equation $\left(7^{\prime}\right)$, we get - for a periodical solution of the type

$$
\dot{x}=-\beta_{0} c \sin \left(\frac{2 \pi \tau}{\tau_{0}}\right) ; \quad \dot{y}=-\beta_{0} c \cos \left(\frac{2 \pi \tau}{\tau_{0}}\right) ; \quad \dot{z}=0
$$

(which describes a uniform circular motion) and by imposing the kinetic energy of the internal rotational motion to equal the intrinsic energy $m_{0} c^{2}$ of the particle - that the amplitude of the oscillations is given by $\beta_{0}^{2}=\frac{3}{4}$. Thus, the magnetic moment corresponding to this motion is exactly the anomalous magnetic moment of the electron, obtained 
in a purely classical context: $\mu_{a}=\frac{1}{4 \pi} \frac{e^{3}}{m_{0} c^{2}}$. This shows, by the way, that the anomalous magnetic moment is an intrinsically classical, and not quantum, result; and the absence of $\hbar$ in the last expression is a confirmation of this fact.

\section{Discretized Quantum Mechanics}

Let us pass to a topic we are more interested in, which is a second step towards our eventual application of the discretization procedures for a possible solution of the measurement problem in Quantum Mechanics, without having to make recourse to the reduction (wavepacket instantaneous collapse) postulate. Namely, let us focus our attention, now, on the consequences for QM of the introduction of a chronon. In our (unpublished) refs. [1], we have extensively examined such consequences: Here, we shall recall only some useful results.

There are physical limits that, even in ordinary QM, seem to prevent the distinction of arbitrarily close successive states in the time evolution of a quantum system. Basically, such limitations result from the Heisenberg relations; in such a way that, if a discretization is to be introduced in the description of a quantum system, it cannot possess a universal value (since those limitations depend on the characteristics of the particular system under consideration): In other words, the value of the fundamental interval of time has to change a priori from system to system. All these points are in favour of the extension of Caldirola's procedure to QM. Time will still be a continuous variable, but the evolution of the system along its world line will be regarded as discontinuous. In analogy with the electron theory in the non-relativistic limit, one has to substitute the corresponding finite-difference expression for the time derivatives; e.g.:

$$
\frac{\mathrm{d} f(t)}{\mathrm{d} t} \rightarrow \frac{f(t)-f(t-\Delta t)}{\Delta t}
$$

where proper time is now replaced by the local time $t$. The chronon procedure can then be applied to obtain the finite-difference form of the Schrödinger equation. As for the electron case, there are three different ways to perform the discretization, and three "Schrödinger equations" can be obtained:

$$
\begin{array}{r}
i \frac{\hbar}{\tau}[\Psi(\mathbf{x}, \mathbf{t})-\Psi(\mathbf{x}, \mathbf{t}-\tau)]=\hat{H} \Psi(\mathbf{x}, \mathbf{t}), \\
i \frac{\hbar}{2 \tau}[\Psi(\mathbf{x}, \mathbf{t}+\tau)-\Psi(\mathbf{x}, \mathbf{t}-\tau)]=\hat{H} \Psi(\mathbf{x}, \mathbf{t}), \\
i \frac{\hbar}{\tau}[\Psi(\mathbf{x}, \mathbf{t}+\tau)-\Psi(\mathbf{x}, \mathbf{t})]=\hat{H} \Psi(\mathbf{x}, \mathbf{t}),
\end{array}
$$


which are, respectively, the retarded, symmetric and advanced Schrödinger equations, all of them transforming into the (same) continuous equation when the fundamental interval of time (that can now be called just $\tau$ ) goes to zero.

Since the equations are different, the solutions they provide are also fundamentally different. As we have already seen, in the classical theory of the electron the symmetric equation represented a non-radiating motion, providing only an approximate description of the motion (without taking into account the effects due to the self fields of the electron). However, in the quantum theory it plays a fundamental role. In the discrete formalism too, the symmetrical equation constitutes the only way to describe a bound non-radiating particle. Let us remark that, for a time independent Hamiltonian, the outputs obtained in the discrete formalism by using the symmetric equation resulted to be [1] very similar to those obtained in the continuous case. For these Hamiltonians, the effect of discretization appears basically in the frequencies associated with the time dependent term of the wave function; and, in general, seem to be negligible.

However, the solutions of the retarded (and advanced) equations show a completely different behaviour. For a Hamiltonian explicitly independent of time, the solutions have a general form given by

$$
\Psi(\mathbf{x}, \mathbf{t})=\left[1+i \frac{\tau}{\hbar} \hat{H}\right]^{-t / \tau} f(\mathbf{x})
$$

and, expanding $f(x)$ in terms of the eigenfunctions of $\hat{H}$ :

$$
\hat{H} u_{n}(\mathbf{x})=W_{n} u_{n}(\mathbf{x})
$$

that is, writing $f(\mathbf{x})=\sum_{n} c_{n} u_{n}(\mathbf{x})$, with $\sum_{n}\left|c_{n}\right|^{2}=1$, one can obtain that

$$
\Psi(\mathbf{x}, \mathbf{t})=\sum_{n} c_{n}\left[1+i \frac{\tau}{\hbar} W_{n}\right]^{-t / \tau} u_{n}(\mathbf{x})
$$

The norm of this solution is given by

$$
|\Psi(\mathbf{x}, \mathbf{t})|^{2}=\sum_{n}\left|c_{n}\right|^{2} \exp \left(-\gamma_{n} t\right)
$$

with

$$
\gamma_{n}=\frac{1}{\tau} \ln \left(1+\frac{\tau^{2}}{\hbar^{2}} W_{n}^{2}\right)=\frac{W_{n}^{2}}{\hbar^{2}} \tau+O\left(\tau^{3}\right),
$$

where it is apparent that the damping factor depends critically on the value $\tau$ of the chronon. This dissipative behaviour originates from the character of the retarded equation; in the case of the electron, the retarded equation possesses intrinsically dissipative solutions, representing a radiating system. The Hamiltonian has the same status as in 
the ordinary (continuous) case: It is an observable, since it is a hermitian operator and its eigenvectors form a basis of the state space. However, as we have seen, the norm of the state vector is not constant any longer, due to the damping factor. An opposite behaviour is observed for the solutions of the advanced equation, in the sense that they increase exponentially.

One of the most impressive achievement due to the introduction of the chronon hypothesis in the realm of QM has been obtained in the description of a bound electron by using the new formalism. In fact, Caldirola found for the excited state of the electron the value $E \simeq 105.55 \mathrm{MeV}$, which is extremely close (with an error of $0.1 \%$ ) to the measured value of the rest mass of the muon. For this, and similar questions, we just refer the reader to the quoted literature.

\section{Discretized (retarded) Liouville equation, and a so- lution of the measurement problem: Decoherence from interaction with the environment}

Suppose we want to measure [6] the dynamical variable $R$ of a (microscopic) object $\mathcal{O}$, by utilizing a (macroscopic) measuring apparatus $\mathcal{A}$. The eigenvalue equation $R|r\rangle_{\mathcal{O}}=r|r\rangle_{\mathcal{O}}$ defines a complete eigenvector-basis for the observable $R$; so that any state $|\psi\rangle_{\mathcal{O}}$ of $\mathcal{O}$ can be given by the expansion $|\psi\rangle_{\mathcal{O}}=\sum_{r} c_{r}|r\rangle_{\mathcal{O}}$.

As to the apparatus $\mathcal{A}$, we are interested only in its observable $A$, whose eigenvalues $\alpha$ represent the value indicated by a pointer; then, we can write $A|\alpha, N\rangle_{\mathcal{A}}=\alpha|\alpha, N\rangle_{\mathcal{A}}$, quantity $N$ representing the set of internal quantum numbers necessary to specify a complete eigenvector-basis for it. Let the initial state of $\mathcal{A}$ be $|0, N\rangle_{\mathcal{A}}$; in other words, the pointer is assumed to indicate initially the value zero. The interaction between $\mathcal{O}$ and $\mathcal{A}$ is expressed by a time - evolution operator $U$, which is expected to relate the value of $r$ with the measurement $\alpha$.

In conventional ("continuous") quantum mechanics, the density operator, $\rho$, obeys the Liouville-von Neumann (LvN) equation

$$
\frac{\mathrm{d} \rho}{\mathrm{d} t}=-\frac{i}{\hbar}[H, \rho] \equiv-i \mathcal{L} \rho(t)
$$

where $\mathcal{L}$ is the Liouville operator; so that, if the hamiltonian $H$ is independent of time, the time evolution of $\rho$ is

$$
\rho\left(t-t_{0}\right)=\exp \left(-\frac{i}{\hbar} H\left(t-t_{0}\right)\right) \rho_{0} \exp \left(\frac{i}{\hbar} H\left(t-t_{0}\right)\right) .
$$


It is known that, if the compound system $\mathcal{O}$ plus $\mathcal{A}$ is initially, for instance, in the mixed state

$$
\rho^{\text {in }}=\sum_{M} C_{M}\left|\psi_{M}^{\text {in }}\right\rangle\left\langle\psi_{M}^{\text {in }}\right|
$$

where quantities $C_{M}$ are (classical) probabilities associated with the states $\left|\psi_{M}^{\text {in }}\right\rangle$, then the "continuous" approach is known to forward

$=\sum_{r_{1}, r_{2}} c_{r_{1}}^{*} c_{r_{2}} \sum_{M} C_{M}\left\{\left|\alpha_{r_{1}} ; r_{1}, M\right\rangle\left\langle\alpha_{r_{2}} ; r_{2}, M\right|\right\}$

$$
\rho^{\mathrm{fin}} \equiv U \rho U^{\dagger}=\sum_{M} C_{M}\left|\psi_{M}^{\mathrm{fin}}\right\rangle\left\langle\psi_{M}^{\mathrm{fin}}\right|=
$$

where the off-diagonal terms yield a coherent superposition of the corresponding eigenvectors. In this case, the reduction postulate has to imply that, in the measurement process, the non-diagonal terms do instantaneously vanish.

On the contrary, in the discrete case, with the interaction embedded in the Hamiltonian $H$, the situation is completely different. Let us consider the energy representation, where $|n\rangle$ are the states with defined energy: $H|n\rangle=E_{n}|n\rangle$. Since the time evolution operator is a function of the Hamiltonian, and commutes with it, the basis of the energy eigenstates will be a basis also for this operator.

The discretized (retarded) Liouville--von Neumann equation is

$$
\frac{\rho(t)-\rho(t-\tau)}{\tau}=-i \mathcal{L} \rho(t)
$$

which reduces to the $\mathrm{LvN}$ equation when $\tau \rightarrow 0$. The essential point is that, following e.g. a procedure similar to Bonifacio's[]], one gets in this case a non-unitary time-evolution operator:

$$
V(t, 0)=\left[1+\frac{i \tau \mathcal{L}}{\hbar}\right]^{-t / \tau},
$$

which, as all non-unitary operators, does not preserve the probabilities associated with each of the energy eigenstates (that make up the expansion of the initial state in such a basis of eigenstates). We are interested in the time instants $t=k \tau$, with $k$ an integer.f

\footnotetext{
${ }^{\dagger}$ By contrast, if we consider as initial state for the system $\mathcal{O}$ plus $\mathcal{A}$ the pure state $\left|\psi_{N}^{\text {in }}\right\rangle=$ $|\psi\rangle_{\mathcal{O}} \otimes|0, N\rangle_{\mathcal{A}} \equiv|\psi\rangle_{\mathcal{O}}|0, N\rangle_{\mathcal{A}}$, then, within the ordinary "continuous" approach, the time evolution leads necessarily to a coherent superposition of (macroscopically distinct) eigenvectors: $U\left(t, t_{0}\right)|\psi\rangle_{\mathcal{O}}|0, N\rangle_{\mathcal{A}}=\sum_{r} c_{r}\left|\alpha_{r} ; r, N\right\rangle \equiv\left|\psi_{N}^{\mathrm{fin}}\right\rangle$. As a consequence, as wellknown, one has to postulate a state collapse from $\left|\psi_{N}^{\text {fin }}\right\rangle$ to $\left|\alpha_{r_{0}} ; r_{0}, N\right\rangle$, where $r_{0}$ is the value indicated by the pointer after the measurement.

${ }^{\ddagger}$ Let us emphasize that the appearance of non-unitary time-evolution operators is not associated with the coarse graining approach only, since they also come out from the discrete Schrödinger equations.
} 
Thus, the time-evolution operator (13) takes the initial density operator $\rho^{\text {in }}$ to a final state for which the non-diagonal terms decay exponentially with time; namely, to

$$
\rho_{r s}^{\mathrm{fin}}=\langle r|V(t, 0)| s\rangle=\rho_{r s}^{\mathrm{in}}\left[1+i \omega_{r s} \tau\right]^{-t / \tau},
$$

where

$$
\omega_{r s} \equiv \frac{1}{\hbar}\left(E_{r}-E_{s}\right) \equiv \frac{1}{\hbar}(\Delta E)_{r s} .
$$

Expression (14) can be written

$$
\rho_{r s}(t)=\rho_{r s}(0) \mathrm{e}^{-\gamma_{r s} t} \mathrm{e}^{-i \nu_{r s} t}
$$

with

$$
\begin{gathered}
\gamma_{r s} \equiv \frac{1}{2 \tau} \ln \left(1+\omega_{r s}^{2} \tau^{2}\right) \\
\nu_{r s} \equiv \frac{1}{\tau} \tan ^{-1}\left(\omega_{r s} \tau\right)
\end{gathered}
$$

One can observe, indeed, that the non-diagonal terms tend to zero with time, and that the larger the value of $\tau$, the faster the decay becomes. Actually, the chronon $\tau$ is now an interval of time related no longer to a single electron, but to the whole system $\mathcal{O}+\mathcal{A}$. If one imagines the time interval $\tau$ to be linked to the possibility of distinguishing two successive, different states of the system, then $\tau$ can be significantly larger than $10^{-23} \mathrm{~s}$, implying an extremely faster damping of the non-diagonal terms of the density operator: See Fig.11.

\section{Comments and Conclusions}

It should be noticed that the time-evolution operator (13) preserves trace, obeys the semigroup law, and implies an irreversible evolution towards a stationary diagonal form. In other words, notwithstanding the simplicity of the present "discrete" theory, that is, of the chronon approach, an intrinsic relation is present between measurement process and irreversibility: Indeed, the operator (13), meeting the properties of a semigroup, does not possess in general an inverse (and non-invertible operators are, of course, related to irreversible processes). For instance, in a measurement process in which the microscopic object is lost after the detection, one is just dealing with an irreversible process that could be well described by an operator like (13).

In our (discrete and retarded) theory, the "state reduction"

$$
\rho(t) \stackrel{t \rightarrow 0}{\rightarrow} \sum_{n} \rho_{n n}(0)|n\rangle\langle n|
$$


Figure 1: Damping of the non-diagonal terms of the density operator for two different values of $\tau$. For both cases we used $\Delta E=4 \mathrm{eV}$. (a) Slower damping for $\tau=6.26 \times 10^{-24} \mathrm{~s}$; (b) faster damping for $\tau=\times 10^{-19} \mathrm{~s}$.

is not instantaneous, but depends - as we have already seen - on the characteristic value $\tau$. More precisely, the non diagonal terms tend exponentially to zero according to a factor which, to the first order, is given by

$$
\exp \left|\frac{-\omega_{n m}^{2} \tau t}{2}\right| .
$$

Thus, the reduction to the diagonal form occurs, provided that $\tau$ possesses a finite value, no matter how small, and provided that $\omega_{n m} \tau$, for every $n, m$, is not much smaller than 1 ; where

$$
\omega_{n m}=\left(E_{n}-E_{m}\right) / \hbar
$$

are the transition frequencies between the different energy eigenstates (the last condition being always satisfied, e.g., for non-bounded systems).

It is essential to notice that decoherence has been obtained above, without having recourse to any statistical approach, and in particular without assuming any "coarse graining" of time. The reduction to the diagonal form illustrated by us is a consequence of the discrete (retarded) Liouville-von Neumann equation only, once the inequality $\omega_{n m} \tau \ll 1$ is not verified.

Moreover, the measurement problem is still controversial even with regard to its mathematical approach: In the simplified formalization introduced above, however, we have not included any consideration beyond those common to the quantum formalism, allowing an as clear as possible recognition of the effects of the introduction of a chronon. 
Let us repeat that the introduction of a fundamental interval of time in the description of the measurement problem made possible a simple but effective formalization of the state reduction process (through a mechanism that can be regarded as a decoherence caused by interaction with the environment [2]) only for the retarded case. This is not obtainable, whem taking into account the symmetric version of the discretized LvN equation.

It may be important to stress that the retarded form (12) of the direct discretization of the $\mathrm{LvN}$ equation is the same equation obtained via the coarse grained description (extensively adopted in [7]). This lead us to consider such an equation as a basic equation for describing complex systems, which is always the case when a measurement process is involved.

Let us add some brief remarks. First: the "decoherence" does not occur when we use the time evolution operators obtained directly from the retarded Schrödinger equation; the dissipative character of that equation, in fact, causes the norm of the state vector to decay with time, leading again to a non-unitary evolution operator: However, this operator (after having defined the density matrix) yields damping terms which act also on the diagonal terms! We discussed this point, as well a the question of the compatibility between Schrödinger's picture and the formalism of the density matrix, have been analyzed by us in an Appendix of our (unpublished) refs. [1]. Second: the new discrete formalism allows not only the description of the stationary states, but also a (space-time) description of transient states: The retarded formulation yields a natural quantum theory for dissipative systems; and it is not without meaning that it leads to a simple solution of the measurement problem in QM. Third: Since the composite system $\mathcal{O}+\mathcal{A}$ is a complex system, it is suitably described by the coarse grained description (exploited by Bonifacio in some important papers of his[7]): it would be quite useful to increase our understanding of the relationship between the two mentioned pictures in order to get a deeper insight on the decoherence processes involved.

\section{Acknowledgments}

One of the authors (ER) wishes to thank Anthony Leggett, Berardo Ruggieri, Paolo Silvestrini, and all the Organizers, for their kind invitation to partecipating in the MQC2 Workshop and to contributing to these Proceedings, as well as for some discussions. For stimulating discussions, or kind collaboration over the years, the authors are moreover very grateful to Rodolfo Bonifacio, Mauro Brambilla, Christian Cocca, Gianni Degli Antoni, Salvatore Esposito, Flavio Fontana, Antony Legget, Giovanni Salesi, Michel Zamboni-Rached, Marisa T. Vasconselos and Daniel Wisniwesky. At last, they acknowledge useful discussions with V.Abate, E.O.Capelas, R.Colombi, G.C.Costa, G.Giuffrida, H.E.Hernández F., L.C.Kretly, P.Leal-Ferreira, F.Pisano, L.Salvo, M.Santini, J.W.Swart, C.Ussami, J.Vaz and M.Villa. 


\section{References}

[1] Farias, Ruy H.A., and Erasmo Recami: "Introduction of a quantum of time (chronon), and its consequences for quantum mechanics", Report IC/98/74 (ICTP; Trieste, 1998), appeared in preliminary form c/o the Lanl Archives as the e-print quant-ph/9706059. Cf. also R.H.A.Farias: "Introduction of a 'quantun' of time into the formalism of quantum mechanics", PhD Thesis, E.Recami supervisor (UNICAMP; Campinas, S.P.; 1994).

[2] See, e.g., H.D.Zee: Found. Phys. 1 (1970) 69; W.H.Zurek: Phys. Rev. D26 (1982) 1862; J.A.Wheeler and W.H.Zurek (eds.): Quantum theory and measurement (Princeton U.P.; Princeton, NJ, 1983); A.O.Caldeira and A.J.Leggett: Physica A121 (1983) 587; Phys. Rev. A31 (1985) 1057; E.Joss and H.D.Zee: Z. Phys. B59 (1985) 223; P.Bush, P.J.Lahti and P.Mittelstaed: The quantum theory of measurement (Springer; Berlin, 1991); W.G.Unruh and W.H.Zurek: Phys. Rev. D40 (1989) 1071; W.H.Zurek: Physics Today 44 (Oct. 1991), p.36; L.Davidovich et al.: Phys. Rev. Lett. 71 (1993) 2360; M.Namiki and S.Pascazio: Phys. Reports 232 (1993) 301; A.J.Leggett: in Foundations of Quantum Mechanics, ed. by M.Tsukada et al. (Tokyo, 1993), p.10; Y.Ne'eman: Phys. Lett. A186 (1994) 5.

[3] P.A.M.Dirac: "The classical theory of electron", Proc. Royal Soc. A167 (1938) 148; Ann. Inst. Poincaré 9 (1938) 13. Cf. also M.Schönberg et al.: Phys. Rev. 69 (1945) 211; and Anais Ac. Brasil. Cie. 19 (1947) issue no.3, pp.46-98.

[4] P.Caldirola: Nuovo Cimento 10 (1953) 1747; Suppl. Nuovo Cim. 3 (1956) 297; Rivista Nuovo Cim. 2 (1979), issue no.13, and refs. therein; Revista Brasil. de Física, special vol. (1984, July), p.228. See also R.Cirelli: Nuovo Cimento 1 (1955) 260; L.Lanz: Nuovo Cimento 23 (1962) 195; F.Casagrande and E.Montaldi: Nuovo Cimento A40 (1977) 369; A44 (1978) 453; P.Caldirola and E.Montaldi: Nuovo Cimento B53 (1979) 291; P.Caldirola, G.Casati and A.Prosperetti: Nuovo Cimento A43 (1978) 127; P.Caldirola: Nuovo Cimento A49 (1979) 497; A.Prosperetti: Nuovo Cimento B57 (1980) 253; L.Belloni: Lett. Nuovo Cim. 31 (1981) 131; V.Benza and P.Caldirola: Nuovo Cimento A62 (1981) 175; G.C.Ghirardi and T.Weber: Lett. Nuovo Cim. 39 (1984) 157; and in particular R.Bonifacio and P.Caldirola: Lett. Nuovo Cim. 38 (1983) 615; 33 (1982) 197. At last, cf. A.D.Yaghjian: Relativistic Dynamics of a Charged Sphere (Springer; Berlin, 1992).

[5] Cf., e.g., E.Recami and G.Salesi: Physical Review A57 (1998) 98; Found. of Phys. 28 (1998) 763; Adv. Appl. Cliff. Alg. 6 (1996) 27; G.Salesi and E.Recami: Found. 
Phys. Lett. 10 (1997) 533; Phys. Lett. A190 (1994) 137; A195 (1994) E389; and refs. therein.

[6] Cf., e.g., L.E.Ballantine: "What is the point of the quantum theory of measurement?", in Fundamental Questions in Quantum Mechanics, ed. by L.Roth \& A.Inomata (Gordon \& Breach; 1986).

[7] See, e.g., R.Bonifacio: Lett. Nuovo Cim. 37 (1983) 481; Nuovo Cimento B114 (1999) 473; Z. für Naturf. A57 (2001) 41. See also R.Bonifacio et al., in [4]. 

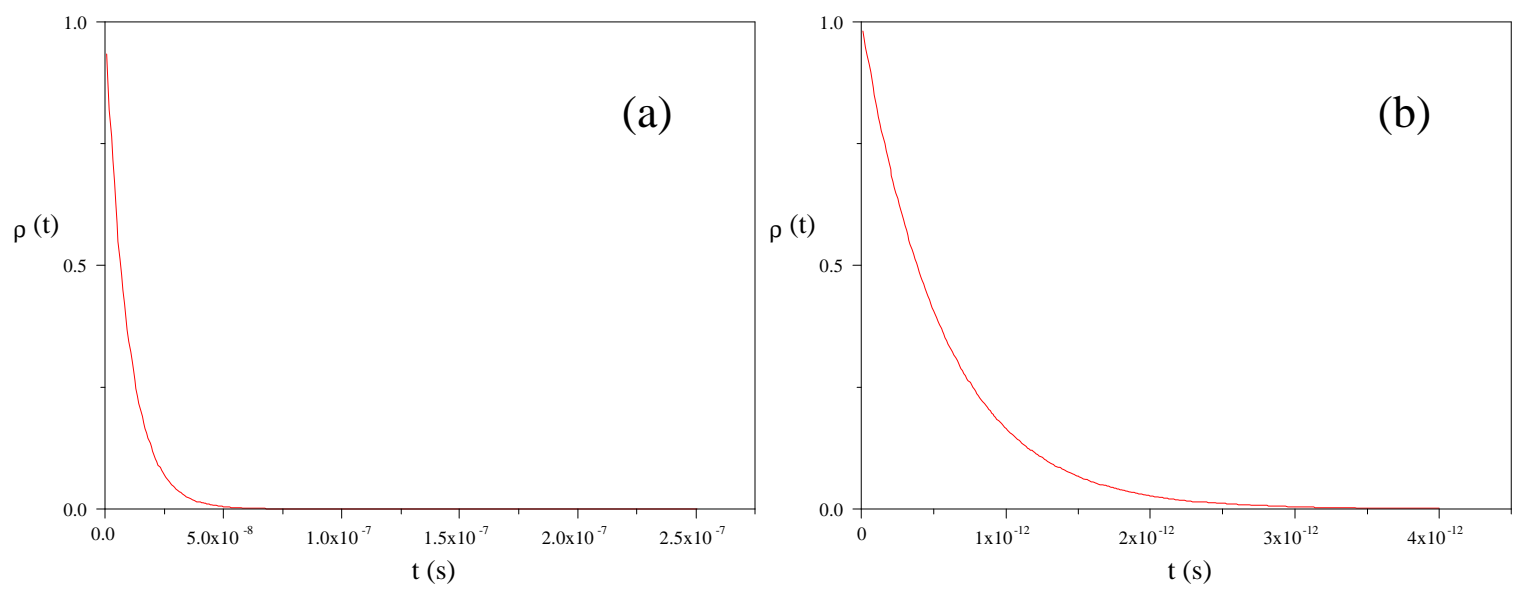